\title{
Synchronization in Dynamically Coupled Fractional-Order Chaotic Systems: Studying the Effects of Fractional Derivatives
}

\author{
J. L. Echenausía-Monroy $\mathbb{D}^{1}{ }^{1}$ C. A. Rodríguez-Martíne $\mathbb{D}^{1},{ }^{1}$ L. J. Ontañón-García $\mathbb{D}^{2}{ }^{2}$ \\ J. Alvarez, ${ }^{1}$ and J. Pena Ramirez $\mathbb{1}^{1}$ \\ ${ }^{1}$ Applied Physics Division, Center for Scientific Research and Higher Education at Ensenada, CICESE. Carr. Ensenada-Tijuana, \\ 3918 Zona Playitas, Ensenada, 22860 BC, Mexico \\ ${ }^{2}$ Coordinación Académica Región Altiplano Oeste, Universidad Autónoma de San Luis Potosí, \\ Carretera a Santo Domingo 200, 78600, Salinas de Hidalgo, S.L.P, Mexico
}

Correspondence should be addressed to J. L. Echenausía-Monroy; jose.luis.echenausia@gmail.com and J. Pena Ramirez; jpena@cicese.mx

Received 27 August 2021; Revised 23 October 2021; Accepted 4 December 2021; Published 24 December 2021

Academic Editor: Atila Bueno

Copyright (c) 2021 J. L. Echenausía-Monroy et al. This is an open access article distributed under the Creative Commons Attribution License, which permits unrestricted use, distribution, and reproduction in any medium, provided the original work is properly cited.

\begin{abstract}
This study presents the effectiveness of dynamic coupling as a synchronization strategy for fractional chaotic systems. Using an auxiliary system as a link between the oscillators, we investigate the onset of synchronization in the coupled systems and we analytically determine the regions where both systems achieve complete synchronization. In the analysis, the integration order is considered as a key parameter affecting the onset of full synchronization, considering the stability conditions for fractional systems. The local stability of the synchronous solution is studied using the linearized error dynamics. Moreover, some statistical metrics such as the average synchronization error and Pearson's correlation are used to numerically identify the synchronous behavior. Two particular examples are considered, namely, the fractional-order Rössler and Chua systems. By using bifurcation diagrams, it is also shown that the integration order has a strong influence not only on the onset of full synchronization but also on the individual dynamic behavior of the uncoupled systems.
\end{abstract}

\section{Introduction}

Synchronization is an emergent physical phenomenon caused by the interaction of two or more dynamic entities that pervade the natural world [1-4]. In the case of oscillating units, the synchronization phenomenon can be defined as the adjustment of temporal evolution to a common rhythm.

For the case of integer-order systems, there exists a vast and mature literature where we can find different interconnection schemes for synchronizing dynamic systems, like, for example, master-slave synchronization scheme, adaptive synchronization, and synchronization based on state observers, to name a few [5-10]. Although each of these strategies is effective, there are limitations in their applications, e.g., there are cases where these schemes have marginal ranges for which the synchronous response is achieved or have poor robustness to maintain a stable synchronous state under the influence of external disturbances. This is one of the reasons why dynamic interconnections have emerged as an alternative to the classical static schemes. In this case, the interaction between agents is indirectly achieved through a suitably designed dynamic coupling [11-13]. This type of synchronization strategy has shown better performance than static couplings. In particular, dynamic coupling increases the intervals of coupling strength values for which it is possible to achieve synchronized behavior, and it may also be possible to synchronize systems that cannot be synchronized with static coupling [11].

On the other hand, the use of fractional calculus has been extensively studied in nonlinear systems (see, e.g., [14-18]) and also, there exist notable contributions related to the study of synchronization in fractional-order systems (see, 
e.g., $[16,19-23])$. For example, there is work based on applying sliding modes to fractional-order models to achieve synchronization [24-29]. The modeling and analytical study of fractional-order systems is also a fruitful field, e.g., the use of the Razumikhin approximation for fractional-order systems with delay [30,31], the extrapolation of Lyapunov theory to fractional systems $[32,33]$, and the existence and uniqueness of equilibrium points of the Mittag-Leffler criteria $[34,35]$. However, the use of dynamic couplings in the context of fractional-order systems seems to be unexplored so far.

Consequently, in this study we present a synchronization scheme of fractional order based on dynamic coupling. In particular, a master-slave interconnection is considered, in which the systems have an indirect interaction via a fractional-order linear system. In the analysis, the Rössler equation [36] and the Chua double-scroll oscillator [37] are considered. Among the questions to be addressed is whether a dynamic coupling designed for integer-order systems is also effective in its fractional-order version? If so, how does the derivative order influence the occurrence of synchronization in the coupled systems? The local stability of the synchronous solution in the coupled systems is investigated by analyzing the error dynamics, and furthermore, the onset of synchronization is also numerically investigated by computing some statistical metrics like Pearson's correlation between time series. Additionally, using bifurcation diagrams, we have investigated the dynamic behavior of the uncoupled systems. The obtained results show that the integration order has a strong influence on the stability of the synchronous solution, and interestingly, it also produces a period-doubling cascade route to chaos in the uncoupled systems.

The rest of the study is organized as follows: Section 2 describes the basics of fractional calculus and gives a brief introduction to fractional-order systems. Then, Section 3 describes the proposed synchronization scheme and the local stability of the synchronous solution in the coupled systems is discussed. Subsequently, in Section 4, the performance of the dynamic coupling is investigated using the Rössler and Chua systems as application examples. Finally, Sections 6 and 7 are dedicated to the discussion and conclusions, respectively.

\section{Preliminaries}

This section presents a brief overview of some basic concepts about fractional-order systems. In particular, the Caputo derivative, the general representation of a fractional-order system, and the stability of linear time-invariant fractionalorder systems are revisited.

2.1. Fractional Caputo Derivative. In the literature, there are various definitions of fractional-order derivatives, the most common being the Riemann-Liouville and Caputo operators $[38,39]$. The fractional Caputo derivative for a timeinvariant system described by the vector field $f(x)$ is defined as

$$
{ }_{a} D_{0}^{q} f(x)=\frac{1}{\Gamma(n-q)} \int_{a}^{x} \frac{f^{(n)}(t)}{(x-t)^{q-n+1}} \mathrm{~d} t,
$$

with $n=\lceil q\rceil$ for the integration order $0<q<1$, being $\Gamma$ the gamma function defined as follows:

$$
\Gamma(z)=\int_{0}^{\infty} t^{z-1} e^{-t} \mathrm{~d} t .
$$

2.2. Fractional-Order Dynamic System. A commensurate fractional-order time-invariant system can be described, in general, as follows:

$$
D_{0}^{n_{k}} x(t)=f\left(t, x(t), D_{0}^{n_{1}} x(t), D_{0}^{n_{2}} x(t), \ldots, D_{0}^{n_{k-1}} x(t)\right),
$$

subject to initial conditions

$$
x^{(j)}(0)=x_{0}^{(j)}, \text { with } j=0,1, \ldots,\left\lceil n_{k}\right\rceil-1,
$$

where $n_{1}, n_{2}, \ldots, n_{k}$ are rational numbers, such that $n_{k}>n_{k-1}>\cdots>n_{1}>0, n_{j}-n_{j-1} \leq 1$ for all $j=2,3, \ldots, k$, and $0<n_{1} \leq 1$. The least common multiple of the denominator of $n_{1}, n_{2}, \ldots, n_{k}$ is defined by $M$ and set $q=1 / M$ and $N=M n_{k}$. Then, equation (3) can be expressed as follows [38]:

$$
\begin{aligned}
D_{0}^{q} x_{0}(t) & =x_{1}(t), \\
D_{0}^{q} x_{1}(t) & =x_{2}(t), \\
& \vdots \\
D_{0}^{q} x_{N-2}(t) & =x_{N-1}(t), \\
D_{0}^{q} x_{N-1}(t) & =f\left(t, x_{0}(t), x_{n_{1} / q}(t), \ldots, x_{n_{k-1} / q}(t)\right) .
\end{aligned}
$$

2.3. Stability of Time-Invariant Fractional-Order System. A linear time-invariant fractional-order system is described by

$$
\frac{\mathrm{d}^{q} x(t)}{\mathrm{d} t^{q}}=A x,
$$

where $x \in \mathbb{R}^{n}$ is the state vector, $A \in \mathbb{R}^{n \times n}$ is a constant matrix, and $0<q<1$ is the fractional commensurate derivative order. Then, the stability of the system described by equation (6) is determined as follows [38]:

(i) System (6) is stable, if and only if $\left|\arg \lambda_{j}\right| \geq q \pi / 2, \forall j=1,2, \ldots, n$

(ii) System (6) is asymptotically stable, if and only if $\left|\arg \lambda_{j}\right|>q \pi / 2, \forall j=1,2, \ldots, n$

(iii) System (6) is unstable, if and only if $\left|\arg \lambda_{j}\right|<q \pi / 2$, for at least one $\lambda_{j}, j=1,2, \ldots, n$

From these conditions, it is clear that the local stability of fractional-order systems depends on the integration order $q$, so that the stability of an equilibrium point can be modified by the fractional order, and therefore, the stability region at the complex plane is as in Figure 1 [40]. 


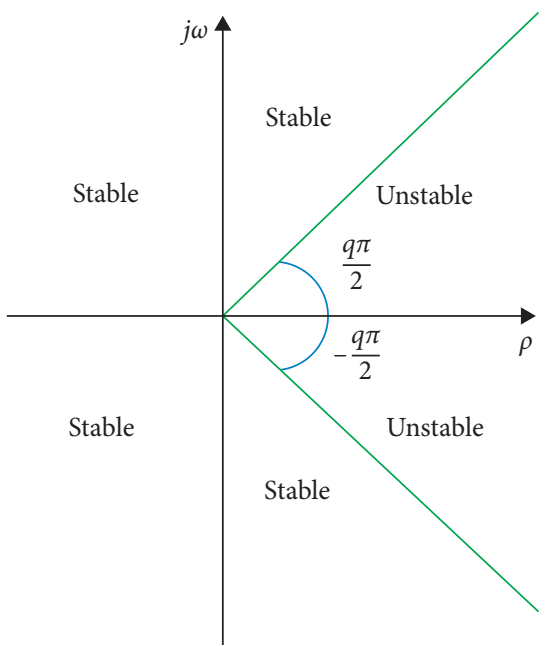

Figure 1: Stability region in a fractional-order linear time-invariant system for $0<q<1$.

\section{Proposed Synchronization Scheme Based on Dynamic Coupling}

The dynamic interconnection considered in this study has been presented in [11] for integer-order systems and is adapted here to the fractional-order case. The scheme, where the interaction between the systems is indirect via a dynamical system (Figure 2) is described by the following set of equations:

$$
\begin{aligned}
D^{q} x_{m} & =F\left(x_{m}\right), \\
D^{q} x_{s} & =F\left(x_{s}\right)-B_{1} h, \\
D^{q} h & =G h-k B_{2}\left(x_{m}-x_{s}\right),
\end{aligned}
$$

where $x_{m}, x_{s} \in \mathbb{R}^{n}$ represents the state vectors of both the master and slave systems and $h=\left(h_{1}, h_{2}\right)^{T}$ for $h_{i} \in \mathbb{R}, i=$ 1,2 is the state variables of the dynamic coupling. It is assumed that the vector field $F$ is smooth enough, which can be either linear or nonlinear, and the coupling force between the systems is denoted by $k$.

On the other hand, the design of a dynamic coupling involves two coupling matrices, denoted $B_{1} \in \mathbb{R}^{n \times 2}$ and $B_{2} \in \mathbb{R}^{2 \times n}$. These matrices are generated under the premise that only one of the elements of each of these matrices is equal to 1 , and the other entries are zero, which means that the coupling is applied only in one state variable of the slave system and that the coupling considers only one measured variable.

Finally, the matrix $G$ from equation (7) is given by

$$
G=\left[\begin{array}{cc}
-\alpha_{c} & 1 \\
-\gamma_{1} & -\gamma_{2}
\end{array}\right],
$$

where $\gamma_{1}, \gamma_{2}$, and $\alpha_{c}$ are design parameters of the dynamic coupling. The construction of the coupling system, for the integer-order case, is inspired by the so-called Huygen's coupling $[11,41]$, which in its simplest form can be interpreted as a damped oscillator.

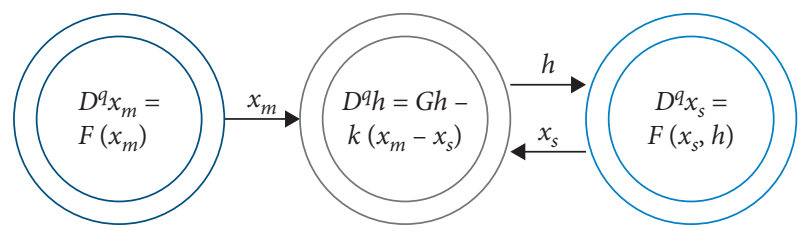

FIGURE 2: Schematic representation of unidirectionally coupled fractional-order systems interacting via fractional-order dynamic coupling.

Since in this study the focus is on synchronization, it is necessary to give the following definition.

Definition 1. The coupled systems, equation (7), are said to be asymptotically synchronized if

$$
\lim _{t \longrightarrow \infty}\left|x_{m}-x_{s}\right|=0, \lim _{t \longrightarrow \infty} h=0 .
$$

3.1. Local Stability Analysis. In order to investigate the stability of the synchronous solution defined in equation (9), we proceed as follows. First, it is assumed that function $F$ in equation (7) can be written as the sum of linear and nonlinear components, i.e., it is assumed that

$$
F\left(x_{i}\right)=P x_{i}+E\left(x_{i}\right),
$$

where $P \in \mathbb{R}^{n \times n}$ is a constant matrix and $E\left(x_{i}\right) \in \mathbb{R}^{n}$ is a vector containing nonlinear terms.

Next, the synchronization error is defined as $e_{p}:=\left(x_{m}-x_{s}, h\right)^{T}$. Note that in the definition of the error, we have included the state $h$ of the dynamic coupling. The reason is because the parameters in the dynamic coupling should be chosen such that, when the systems synchronize, the coupling vanishes. Then, by replacing equations (10) into (7), and computing the corresponding synchronization error dynamics, we obtain

$$
D^{q} e_{p}=\widetilde{A} e_{p}+g_{p}\left(t, e_{p}\right),
$$

where

$$
\begin{aligned}
\widetilde{A} & =\left[\begin{array}{cc}
P & B_{1} \\
-K B_{2} & G
\end{array}\right], \\
g_{p}\left(t, e_{p}\right) & =\left[\begin{array}{c}
f\left(x_{m}\right)-f\left(x_{s}\right) \\
O
\end{array}\right],
\end{aligned}
$$

where $O=(0,0)^{T}$. Furthermore, note that the term $g_{p}\left(t, e_{p}\right)$ is a vanishing perturbation [42] because $g_{p}(t, 0)=0$. Then, the stability properties of system equation (11) are fully determined by the eigenvalues of the matrix $\widetilde{A}$. In particular, following the results presented in Section 2.3, we have that the synchronization error dynamics (equation (11)) is locally asymptotically stable if

$$
\left|\arg \lambda_{j}\right|>\frac{q \pi}{2}, \forall j=1,2, \ldots, n .
$$


Thus, if it is possible to find values of $k, \gamma_{1}, \gamma_{2}$, and $\alpha_{c}$ such that the above condition is satisfied, then the coupled systems described by equation (7) will achieve complete synchronization, according to Definition 1.

3.2. Statistical Metrics Used for Detecting Complete Synchronization. In this study, the onset of synchronization in the coupled systems (equation (7)) is also numerically studied by computing the following synchronization index.

$$
S=\left[\sum_{i=1}^{n}\left|e_{i}\right|+\sum_{i=1}^{n} C_{i}\right]
$$

where $n$ is the dimension of the systems to be synchronized, $\left|e_{i}\right|$ is the absolute value of the synchronization error between the $i$-th state variable of the master and slave systems, i.e.,

$$
\left\langle e_{i}\right\rangle=\left|x_{m i}-x_{s i}\right|,
$$

and $C_{i}$ is the Pearson correlation [43], computed from the $i$-th state variable of the master and slave systems described by

$$
C_{i}=\frac{\sigma_{N_{m i} N_{s i}}}{\sigma_{N_{m i}} \sigma_{N_{s i}}}
$$

where $\sigma_{N_{m i} N_{s i}}$ is the covariance between the data obtained from the time series of the state variables of the master and slave systems, and $\sigma_{N_{m i}} \sigma_{N_{s i}}$ is the standard deviation obtained from the $i$-th state variable of the master (slave) oscillator. Finally, when $S=n$, the systems are synchronized.

In the next section, the onset of synchronization with dynamical coupling is studied for two particular fractionalorder chaotic systems, namely the Rössler and the Chua oscillators.

\section{Application Example 1: Rössler System}

The fractional-order version of the well-known Rössler system [44] is given by

$$
\begin{aligned}
& D^{q} x_{1}=-x_{2}-x_{3}, \\
& D^{q} x_{2}=x_{1}+a x_{2}, \\
& D^{q} x_{3}=b+x_{1} x_{3}-c x_{3},
\end{aligned}
$$

where $x_{i}, i=1,2,3$ denotes the state variables and $a, b, c$ are constants.

It has been reported that every system has a limit of integration order for which it is possible to use a fractionalorder derivative without stabilizing its dynamics $[16,17,45]$. This could be interpreted to mean that the dynamics described in equation (17) are restricted to have at least one eigenvalue in the unstable region, and this being true only if $|\arg (\lambda)|<q \pi / 2$ for at least one of its eigenvalues $(\lambda)$. With $a=0.2, b=0.2, c=5.7$, the eigenvalues obtained by the Jacobian matrix evaluated at the equilibrium point $E_{1}=$ $(0.007,-0.0351,0.0351) \quad$ are $\quad \lambda=[0.0970 \pm 0.9952 i ;-$ 5.6870]. Since $|\min (\arg (\lambda))|=1.4736$, the critical order of integration is defined as $q_{c}=|\min (\arg (\lambda))|(2 / \pi)$ (Section
2 ), and by substitution it is obtained as $q_{c}=0.9381$. This result is confirmed by the bifurcation diagram as shown in Figure 3, where the local maxima in $x_{1}$ are plotted as a function of the variation of the integration order. Note that the derivative order is the same for all state variables of the system. The inset shows the attractors and the corresponding integration order considered for the developed analysis.

It is worth noting that the bifurcation diagram shown in Figure 3 was numerically calculated using the Adams-Bashforth-Moulton (ABM) method [46] and following the guides for plotting a proper bifurcation diagram [47].

4.1. Dynamically Coupled Fractional-Order Rössler Systems. Now, we consider a pair of identical Rössler systems [44] described by

$$
\begin{aligned}
& \text { master system }\left\{\begin{array}{l}
D^{q_{m}} x_{1 m}=-x_{2 m}-x_{3 m}, \\
D^{q_{m}} x_{2 m}=x_{1 m}+a x_{2 m}, \\
D^{q_{m}} x_{3 m}=b+x_{1 m} x_{3 m}-c x_{3 m},
\end{array}\right. \\
& \text { slave system }\left\{\begin{array}{l}
D^{q_{s}} x_{1 s}=-x_{2 s}-x_{3 s}-h_{2}, \\
D^{q_{s}} x_{2 s}=x_{1 s}+a x_{2 s}, \\
D^{q_{s}} x_{3 s}=b+x_{1 s} x_{3 s}-c x_{3 s},
\end{array}\right.
\end{aligned}
$$

dynamic coupling $\left\{\begin{array}{l}D^{q_{d}} h_{1}=-\alpha_{c} h_{1}+h_{2}, \\ D^{q_{d}} h_{2}=-\gamma_{1} h_{1}-\gamma_{2} h_{2}-k\left(x_{2 m}-x_{2 s}\right),\end{array}\right.$

where $x_{m, s}$ denotes the state variables of the master and slave systems, respectively, $h_{1,2}$ denotes the states of the dynamic coupling, $k$ indicates the coupling force between the oscillators, $a, b, c$ are constants of the Rössler model, and $q_{j}, j=$ $m, s, d$ denotes the integration order of the master, slave, and dynamic coupling, respectively.

Here, we consider the case in which all orders of integration are equal, i.e., $q_{m}=q_{s}=q_{d}$. Then, the dynamic behavior of system equations (18)-(20) is numerically studied as a function of the coupling strength $k$ and the integration order $q$. For this purpose, equations (18)-(20) are numerically integrated with the following parameter values cf. [11]: $\quad \alpha_{c}=a, \gamma_{1}=k, \gamma_{2}=k, a=0,2, b=0,2, c=5,7$ and initial conditions $\left[x_{i m}, x_{i s}, h_{i}\right]=[0.1,0.1,0.1,-0.2$, $-0.1,-0.1,0,0]$. On the other hand, the coupling strength is varied in the interval $0 \leq k \leq 20$ with a test size $\Delta_{k}=0.2$ and the integration order is varied in the interval $0.96 \leq q \leq 1$ at $\Delta_{q}=2 e^{-4}$

The obtained results are shown in Figure 4(a), where the colors indicate the value of the synchronization index $S$ (see equation (14)). Synchronous behavior is indicated by the dark blue color $(S=3)$, while the remaining colors indicate unsynchronized dynamics.

Moreover, it is evident from Figure 4(a) that there are regions where, for a fixed coupling strength, the limit behavior is completely determined by the integration order. For example, for a fixed coupling strength of $k=10$, the synchronization index $S$ abruptly changes when the 


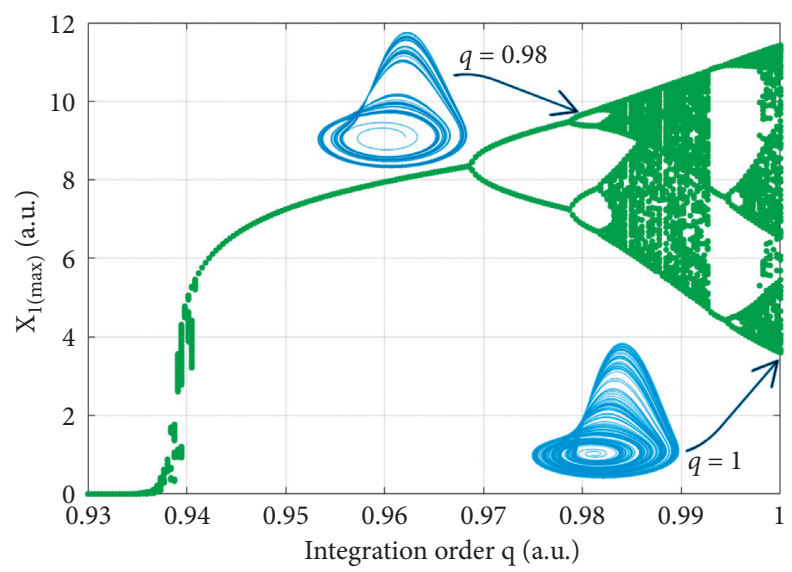

FIgURE 3: Bifurcation diagram of the Rössler system by modifying the integration order, equation (16). The abscissa axis indicates that the three oscillator state variables have the same integration order.

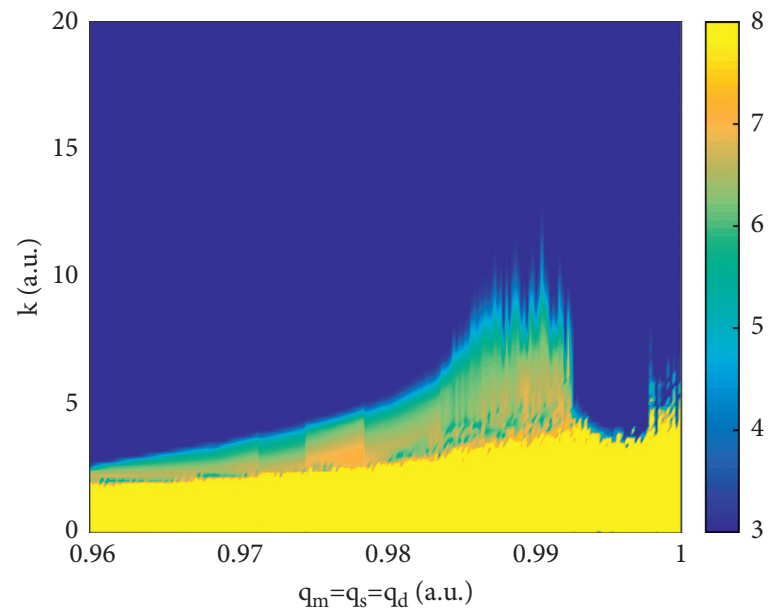

(a)

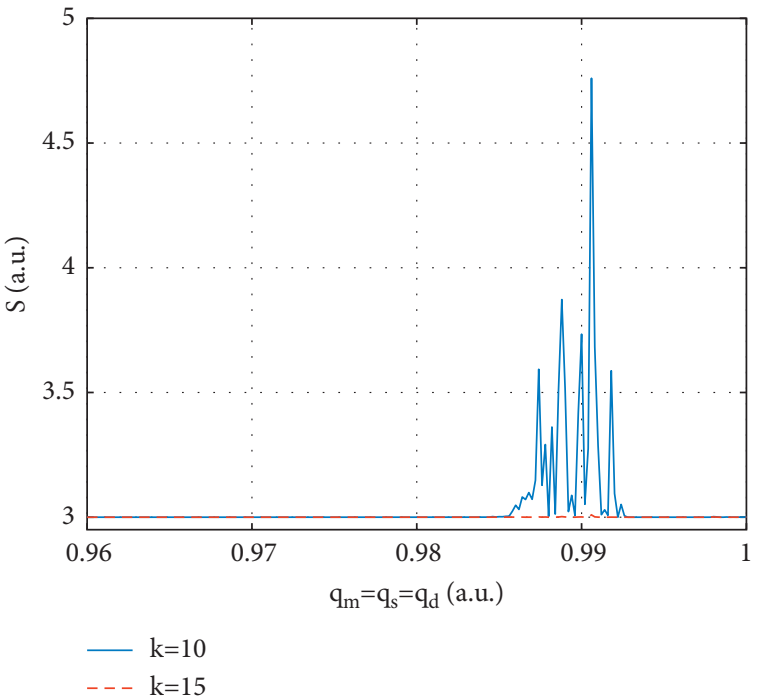

(b)

Figure 4: Numerical results for equations (18)-(20). (a) Limit behavior of the system as a function of coupling strength $k$ and integration order $q$. (b) Synchronization index equation (14) as a function of integration order, for a fixed $k=10$ and $k=15$. A value of $S=3$ indicates complete synchronization.

integration order $q$ is within the interval $0.988<q<0.995$, as can be seen in Figure 4(b), but if the coupling strength is increased up to $k=15$, the systems always achieve complete synchronization.

To validate the synchronization regions obtained by the time series analysis, we conduct a stability analysis following the results presented in Section 3.1. First, let the following synchronization errors be defined as follows: $e_{j}=x_{j m}-x_{j s}$, for $j=1,2,3$, and $e_{4}=h_{1}, e_{5}=h_{2}$. Then, the resulting error dynamics are given by

$$
\begin{aligned}
& D^{q} e_{1}=-e_{2}-e_{3}+e_{5}, \\
& D^{q} e_{2}=e_{1}+a e_{2}, \\
& D^{q} e_{3}=x_{1 m} x_{3 m}-x_{1 s} x_{3 s}-c e_{3}, \\
& D^{q} e_{4}=-\alpha_{c} e_{4}+e_{5}, \\
& D^{q} e_{5}=-\gamma_{1} e_{4}-\gamma_{2} e_{5}-k e_{2} .
\end{aligned}
$$

Note that the error dynamics in equation (21) can be written in the form of equation (11) with 


$$
\begin{aligned}
\widetilde{A} & =\left[\begin{array}{ccccc}
0 & -1 & -1 & 0 & 1 \\
1 & a & 0 & 0 & 0 \\
0 & 0 & -c & 0 & 0 \\
0 & 0 & 0 & -\alpha_{c} & 1 \\
0 & -k & 0 & -\gamma_{1} & -\gamma_{2}
\end{array}\right], \\
g_{p}\left(t, e_{p}\right) & =\left[\begin{array}{c}
0 \\
0 \\
x_{1 m} x_{3 m}-x_{1 s} x_{3 s} \\
0 \\
0
\end{array}\right] .
\end{aligned}
$$

Note that the term $g_{p}\left(t, e_{p}\right)$ in equation (21) is indeed a vanishing perturbation since when the systems synchronize it follows that $x_{1 m}=x_{1 s}, x_{2 m}=x_{2 s}$, and $x_{3 m}=x_{3 s}$ and therefore $g_{p}(t, 0)=0$. Consequently, the local stability of the synchronous solution in the coupled systems described by equations (18)-(20) can be determined from the condition in equation (13). In particular, we compute condition equation (13) as a function of the integration order $q$ and the coupling strength $k$. The obtained results are shown in Figure 5(a), where the blue region corresponds to values of $k$ and $q$ for which condition (13) is not satisfied and thus the synchronous solution is unstable, whereas on the white region, condition (13) is satisfied and then the synchronous solution is expected to be stable. For the sake of comparison, Figure 5(b) shows the overlap of Figure 4 with Figure 5(a). It can be seen that there is a good agreement between the numerical and the analytical results.

Remark 1. In the previous analysis, we have considered the case where the integration order of the systems and the dynamic coupling are the same. However, we also have conducted a numerical study in which the integration orders are different. In particular, we have numerically integrated equations (18)-(20) using the parameter values considered before, as a function of the integration orders of the master and slave systems, while the integration order of the dynamic coupling remains fixed. The integration orders $q_{m}, q_{s}$ are varied in the interval $0.98 \leq q_{j}<1, j=m, s$, and considering the integration order $q_{d}=0.985$. The obtained results are shown in Figure 6(a) where the blue areas correspond to synchronization $(S=3)$. From the obtained results, it is clear to see that the integration order of the master and slave systems should be almost the same to observe a synchronized behavior and that larger differences are tolerated as long as the integration order of both systems approaches to one.

On the other hand, Figure 6(b) shows the obtained results for the case that only the integration order of the dynamic coupling is varied, while the oscillators are assumed to have integer order, i.e., $q_{m}=q_{s}=1$. In this case, equations (18)-(20) are numerically integrated by varying the integration order $q_{d}$ of the dynamic coupling in the interval $0.8<q_{d}<1$ and the corresponding synchronization index, given by equation (14), is calculated. The obtained results are shown in Figure 6(b) for two different coupling forces, where the choice of these $k$ values corresponds to those reported in [11] and those obtained in Figure 4(b).

\section{Application Example 2: Chua System}

If the Chua system described in [37] is modeled with derivatives of fractional order, then the system described in equation (23) is analyzed.

$$
\begin{aligned}
& D^{q} x_{1}=\sigma\left(x_{2}-x_{1}-\phi\left(x_{1}\right)\right), \\
& D^{q} x_{2}=x_{1}-x_{2}+x_{3} \\
& D^{q} x_{3}=-\beta x_{2}
\end{aligned}
$$

where $D^{q}$ is the fractional-order derivative by Caputo's definition, $x_{i}, i=1,2,3$ denotes the system state variables, $\sigma, \beta$ are constants of the Chua circuit, and $\phi\left(x_{1}\right)$ is a nonlinear function defined in equation (24), with constant values $a, b$.

$$
\phi\left(x_{1}\right)=b x_{1}+\frac{1}{2}(a-b)\left(\left|x_{1}+1\right|-\left|x_{1}-1\right|\right) .
$$

In the same way as for the Rössler fractional-order model, the system described in (23) is analyzed to identify the minimum fractional order that can be modeled without stabilizing the dynamics, namely, $q_{c}=0.9541$, since $\sigma=10, \beta=14.87, a=-1.27, b=0.68$. This result is confirmed by the bifurcation diagram as shown in Figure 7 , where the local maxima in $x_{1}$ are plotted as a function of the integration order variation. The inset shows the attractors and the corresponding integration order.

5.1. Dynamically Coupled Fractional-Order Chua Systems. In the same way as for the Rössler system described above, a pair of Chua oscillators [48] coupled by dynamical connections and defined by the system of equations (25)-(27) is considered.

$$
\begin{aligned}
& \text { master system }\left\{\begin{array}{l}
D^{q_{m}} x_{1 m}=\sigma\left(x_{2 m}-x_{1 m}-\phi\left(x_{1 m}\right)\right) \\
D^{q_{m}} x_{2 m}=x_{1 m}-x_{2 m}+x_{3 m} \\
D^{q_{m}} x_{3 m}=-\beta x_{2 m}
\end{array}\right. \\
& \text { slave system }\left\{\begin{array}{l}
D^{q_{s}} x_{1 s}=\sigma\left(x_{2 s}-x_{1 s}-\phi\left(x_{1 s}\right)\right) \\
D^{q_{s}} x_{2 s}=x_{1 s}-x_{2 s}+x_{3 s} \\
D^{q_{s}} x_{3 s}=-\beta x_{2 s}-h_{1},
\end{array}\right. \\
& \text { dynamic coupling }\left\{\begin{array}{l}
D^{q_{d}} h_{1}=-\alpha_{c} h_{1}+h_{2}, \\
D^{q_{d}} h_{2}=-\gamma_{1} h_{1}-\gamma_{2} h_{2}-k\left(x_{3 m}-x_{3 s}\right)
\end{array}\right.
\end{aligned}
$$

where $x_{m, s}$ denotes the state variables of the master and slave systems, respectively, $h_{1,2}$ is the states of the dynamic coupling, $\phi\left(x_{1 m, s}\right)$ is the nonlinear function defined in equation (28), and $k$ is the coupling force between the systems. 


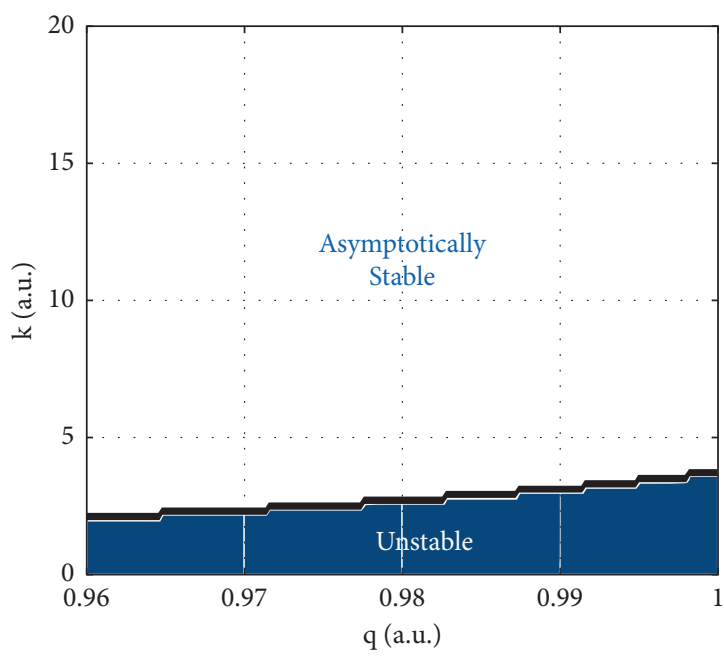

(a)

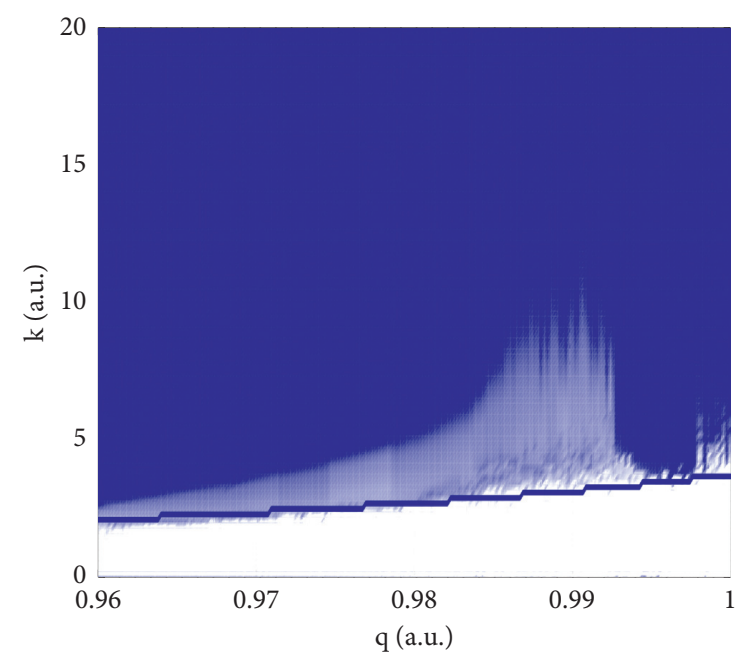

(b)

FIGURE 5: (a) Stability analysis from linear error dynamics for the coupled Rössler system shown in equation (21). (b) Overlap of the analytical result in the synchronization map shown in Figure 4(a).

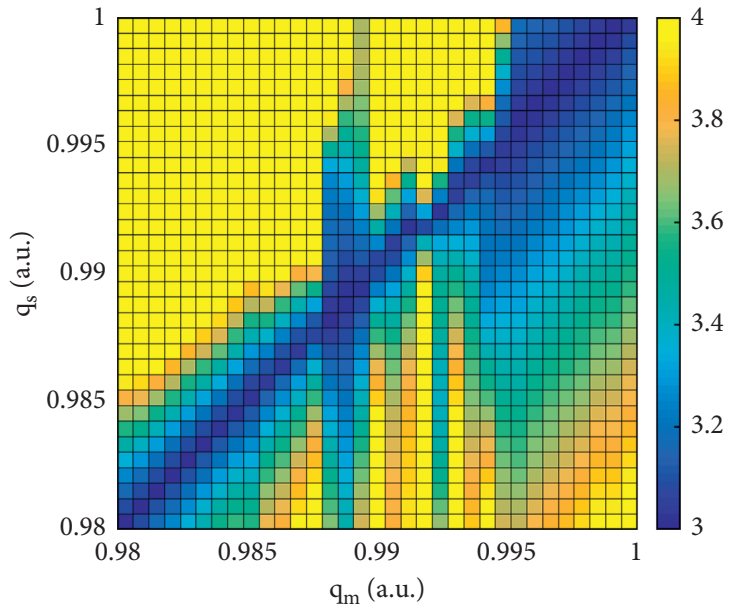

(a)

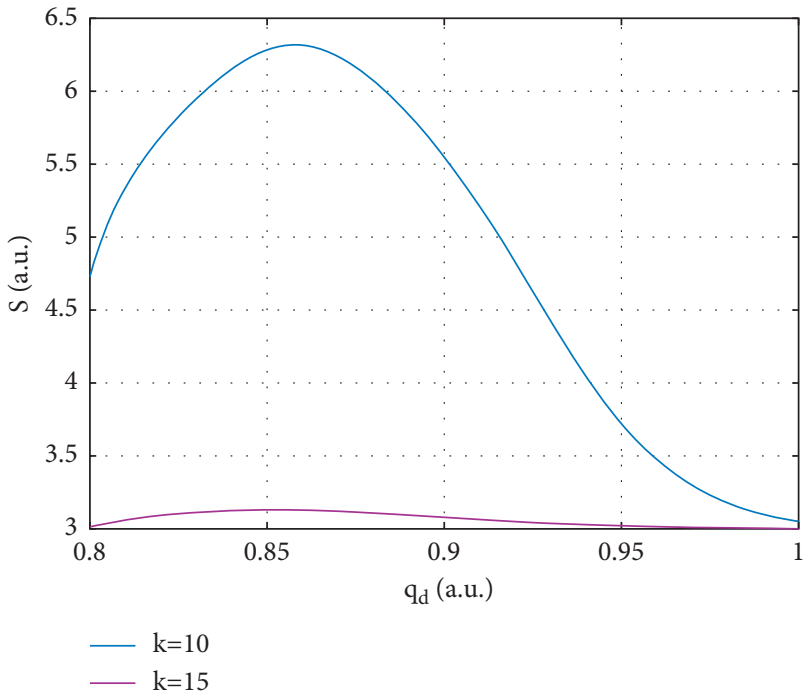

(b)

FIgURE 6: (a) Synchronization map as a function of integration orders $q_{m}$ and $q_{s}$ of master and slave Rössler systems, equations (18)-(20), and considering a fixed integration order of $q_{d}=0.985$ in the dynamic coupling. (b) Synchronization map for system equations (18)-(20) as a function of the integration order $q_{d}$ of the dynamic coupling, while considering that the master and slave oscillators have fixed integer order, i.e., $q=1$.

$$
\phi\left(x_{1 i}\right)=b x_{1 i}+\frac{1}{2}(a-b)\left(\left|x_{1 i}+1\right|-\left|x_{1 i}-1\right|\right), \text { for } i=m, s .
$$

As reported in [11], the following values are used in this study for all analyzes developed; $\sigma=10, \beta=14.87, a=$ $-1.27, b=-0.68$ for $\alpha_{c}=1, \gamma_{1}=k, \gamma_{2}=k / 5$, and initial conditions $\left[x_{i m}, x_{i s}, h_{i}\right]=[0.1,0.1,0.1,-0.2,-0.1,-0.1,0,0]$. On the other hand, the coupling strength is varied in the interval $0 \leq k \leq 60$ with $\Delta_{k}=0.5941$ and the integration order is varied in the interval $0.965 \leq q \leq 1$ for step size.
To identify synchronization regions in the coupled Chua systems of fractional order, an analysis of the coupling force as a function of the integration order is developed. The obtained results are shown in Figure 8. Note that the color map represents the value of the metric $S$ and that the darkest shade of blue represents $S=3$, which means that the systems have reached full synchronization.

Analogous to the stability analysis performed for the coupled Rössler system, the dynamic error model of the coupled pair of Chua oscillators described in equations (25)-(27) is described by 


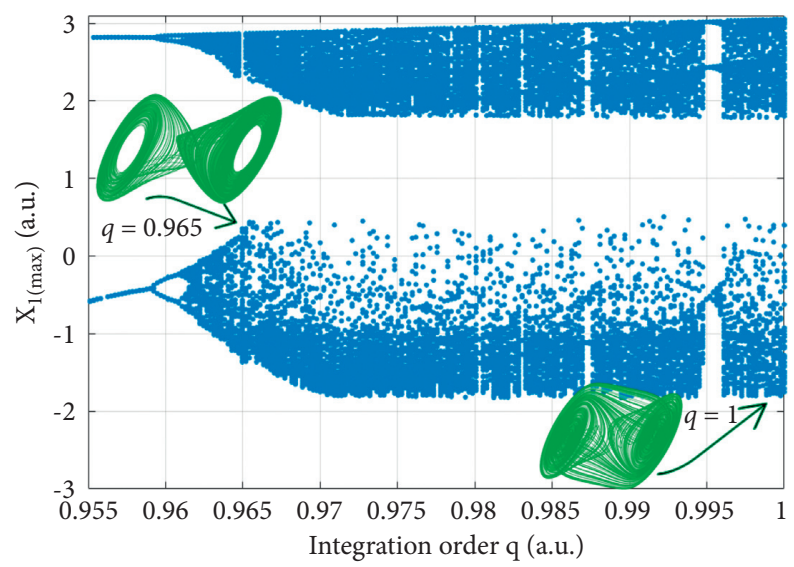

FIGURE 7: Bifurcation diagram of the Chua system by modifying the integration order, equation (23). The abscissa axis indicates that the three oscillator state variables have the same integration order.

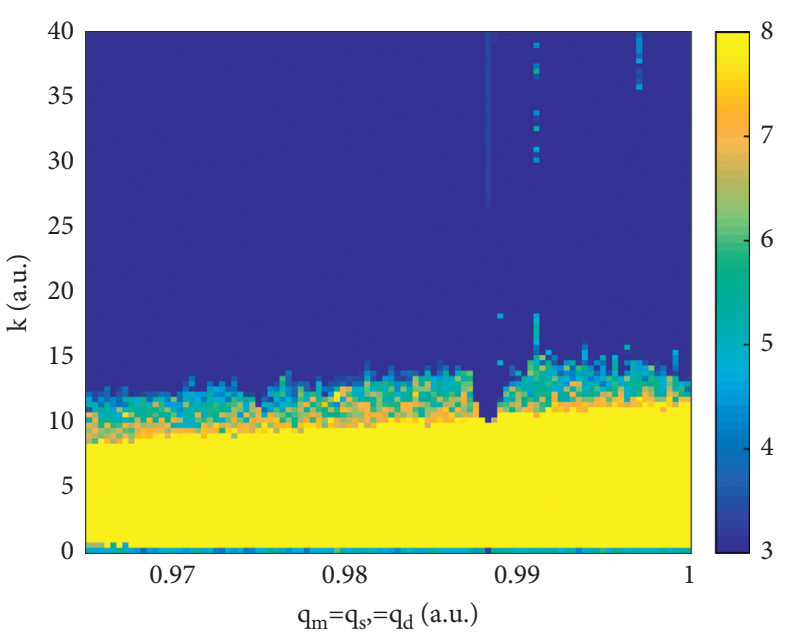

(a)

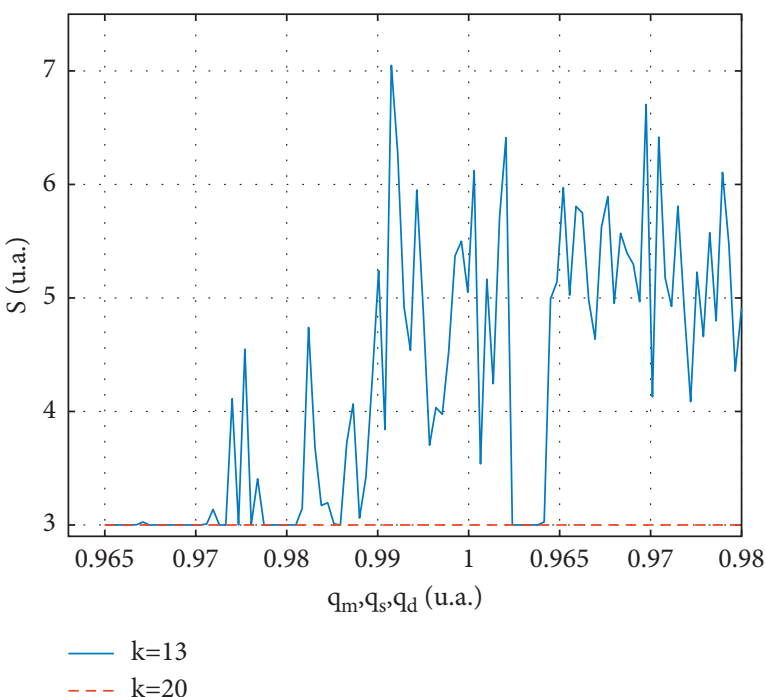

(b)

FigURE 8: Numerical result equations (25)-(27). (a) Limit behavior of the system as a function of coupling strength $k$ and integration order $q$. (b) Synchronization index equation (14) as a function of integration order, for a fixed $k=13$ and $k=20$. A value of $S=3$ indicates complete synchronization.

$$
\begin{aligned}
& D^{q} e_{1}=\sigma\left(e_{2}-e_{1}-\left[\phi\left(x_{1 m}\right)-\phi\left(x_{1 s}\right)\right]\right), \\
& D^{q} e_{2}=e_{1}-e_{2}+e_{3}, \\
& D^{q} e_{3}=-\beta e_{2}-h_{1}, \\
& D^{q} e_{4}=-\alpha e_{4}+e_{5}, \\
& D^{q} e_{5}=-\gamma_{1} e_{4}-\gamma_{2} e_{5}-k e_{3} .
\end{aligned}
$$

Due to the nature of the nonlinearity of the Chua circuit, it is not possible to perform the same analysis as in the Rössler system; instead, it is necessary to use the Jacobian of the error model, equation (30), evaluated in one of the equilibrium points of the system. Since the Chua circuit has symmetric equilibrium points located at $E_{1}=(-1.841$, $0.0004474,2.179)$ for the previously defined values, the choice of one of these points does not affect the analysis.

$$
\widetilde{J}=\left[\begin{array}{ccccc}
-\sigma-\sigma p(x) & \sigma & 0 & 0 & 0 \\
1 & -1 & 1 & 0 & 0 \\
0 & -\beta & 0 & 1 & 0 \\
0 & 0 & 0 & -\alpha_{c} & 1 \\
0 & 0 & 0 & -\gamma_{1} & -\gamma_{2}
\end{array}\right] \text {, }
$$

where $p(x)=1 / 2(b \operatorname{sign}(|x|-1)+b)-1 / 2(a \operatorname{sign}(|x|-1)$ $-a)$ ).

After defining the system shown in equation (30), it is possible to perform the stability system analysis, where the stability of the dynamic model of the Chua coupling error is sought by modifying the integration order and the coupling force. The analytical result is shown in Figure 9(a) and then compared with the map obtained from the time series 


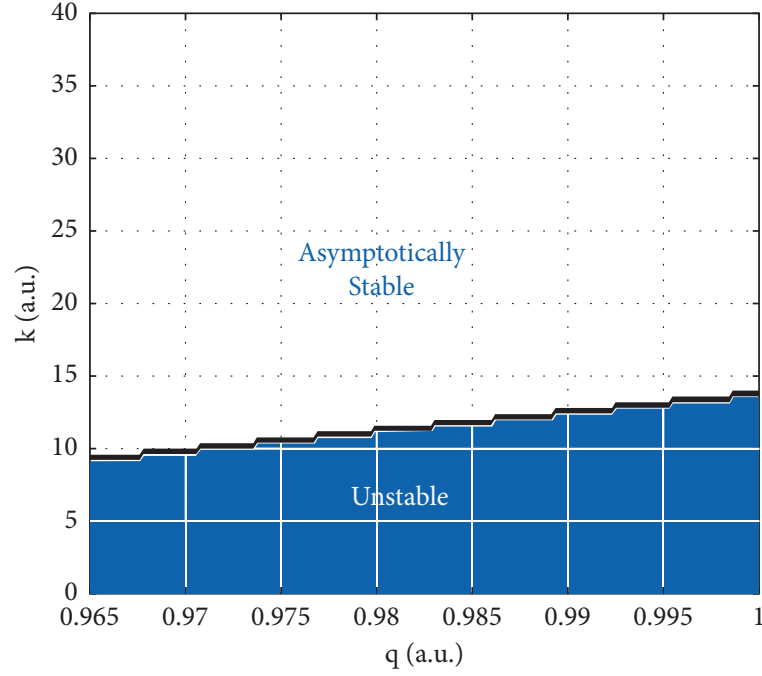

(a)

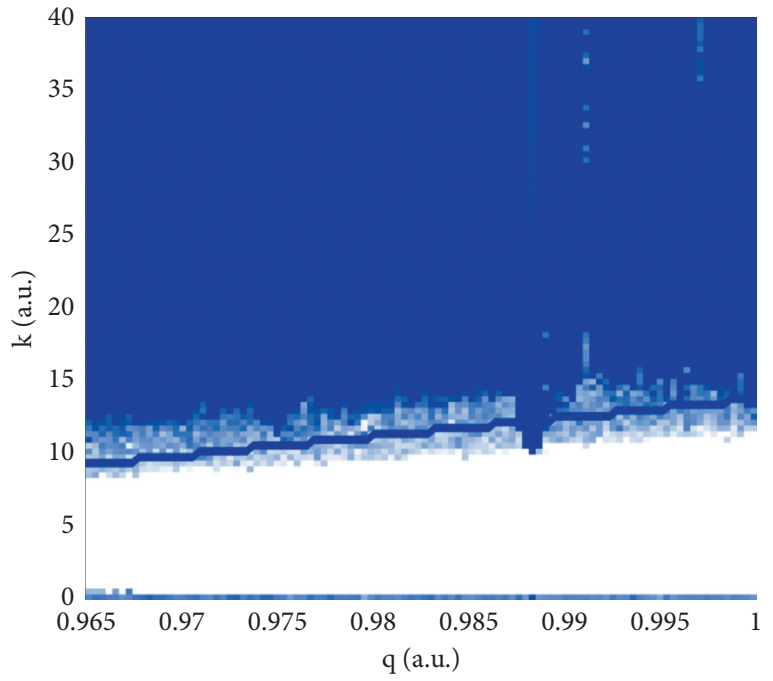

(b)

Figure 9: (a) Stability analysis from linear error dynamics for the coupled Chua system as shown in equation (29). (b) Overlay of the analytical result in the synchronization map as shown in Figure 8(a).

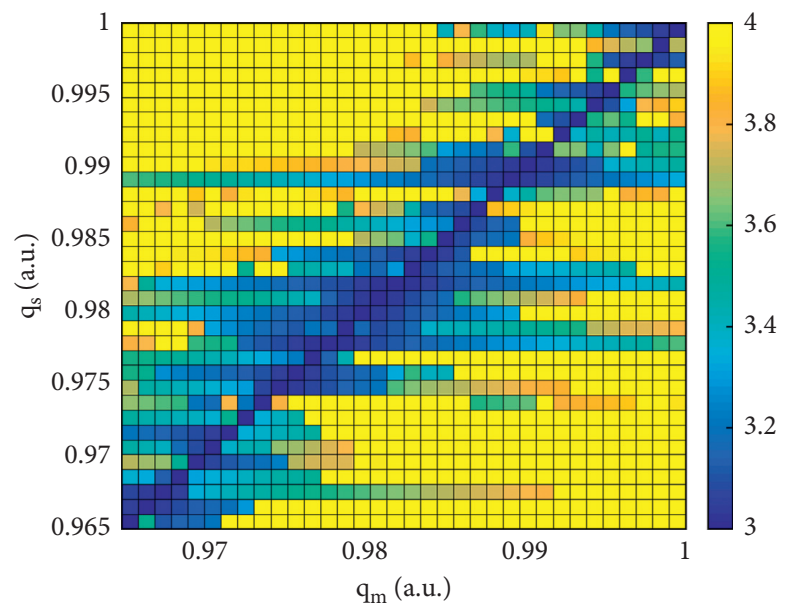

(a)

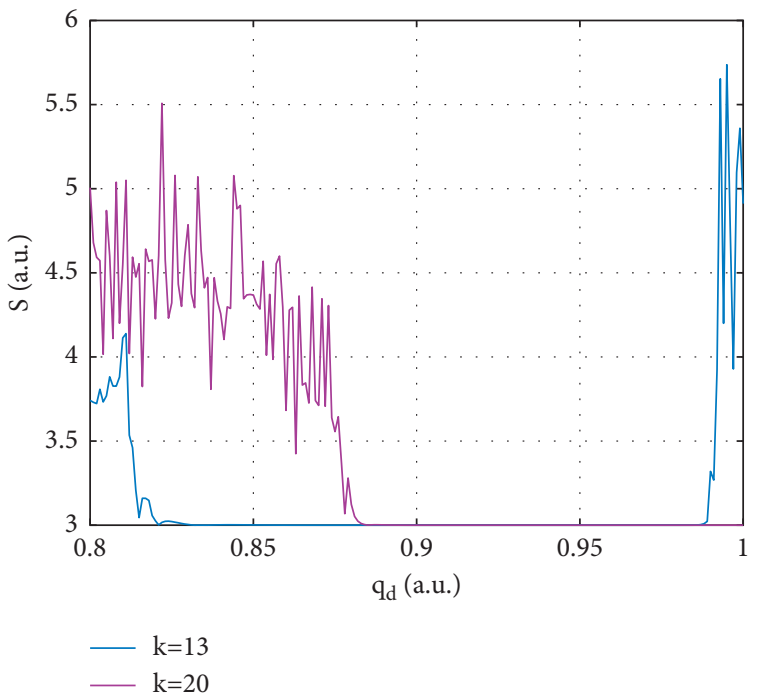

(b)

FIGURE 10: Synchronization map as a function of integration orders for the Chua systems, equations (25)-(27). (a) The integration order of the master and slave is varied, whereas the integration order of the dynamic coupling remains fixed. (b) The order of the dynamic coupling is varied, while the master and slave systems have fixed integer order $q=1$.

analysis in Figure 8(b). As with the Rössler case, the analytical result is able to describe the boundary at which the system is unstable.

Remark 2. Similarly, to the case of the Rössler systems, we have also investigated the onset of synchronization in the fractional-order Chua oscillators as a function of the derivative orders in the oscillators while keeping the coupling system at fixed $q_{d}$, resulting in the map as shown in Figure 10(a). The results from the analysis of the behavior of the Chua systems under the dynamic coupling integration order variation are shown in Figure 10(b), where the integration orders of the oscillators remain fixed at $q_{m, s}=1$.

\section{Discussion}

From the results presented in this study, it is possible to confirm the research question formulated in the introduction of this study, according to which the use of dynamic couplings in chaotic systems of fractional order is able to induce complete synchronization, as it has been reported in their counterparts of integer order. Likewise, it is noteworthy 
to mention that the transition from unsynchronized behavior to synchronization is not abrupt, since there exists a region where the coupled systems may exhibit some sort of intermittency phenomenon. These areas are indicated by the blurred areas in Figures 4 and 8.

If the oscillators are modeled with fractional derivatives but a fixed integration order is maintained in the coupling system, the desired synchronous behavior is achieved only under the condition that both oscillators have the same integration order. Small variations in the integration order in some of the models cause the systems to lose their synchrony, as shown in Figures 6(a) and 10(a). In contrast, the synchronization seems to have some robustness against variations in the integration order of the dynamic coupling, provided that the oscillators have the same integration order, as shown in the numerical results presented in Figures 6(b) and $10(\mathrm{~b})$.

It is also noteworthy that in the $(q, k)$ plane there exist regions where the systems are easier to synchronize. This is explained by the bifurcation diagrams of the isolated oscillators, given in Figures 3 and 7, where the modification in the integration order causes important qualitative changes in the dynamics of the system, where both models are able to present chaotic or periodic behavior, for a set of parameters where the integer-order dynamics is always chaotic, only due to the modification of the derivative order. This is not only an indication that the synchronization between the systems requires lower coupling forces for periodic and quasiperiodic behaviors but also a clear indication that the change in the integration order can be associated with a modification of the vector field, which can also be achieved in the integerorder system by modifying the system parameters [17].

The local stability analysis is in good agreement with the numerical analysis, as shown in Figures 5 and 9. It should be noted, however, that the stability conditions are only necessary conditions. This result is similar when talking about the stability of the equilibrium points in a chaotic system, where obtaining unstable saddle points with index 2 favors the occurrence of chaotic behavior but does not guarantee the occurrence of a strange attractor in the system [49].

It is worth mentioning that in the cases of analysis where the equations to solve do not have the same derivative order, the algorithm proposed by Petráš is implemented [50]; otherwise, the Adams-Bashforth-Moulton (ABM) method is used [46], which is a generalization of the classical ABM integrator that is well known in the resolution of first-order switching system problems $[40,51]$.

Notice that the results presented here have been obtained under the assumption of identical oscillators. It is still necessary to determine the robustness of the dynamic coupling against parameter mismatches or external disturbances in the oscillators.

\section{Conclusions}

We have analyzed the onset of synchronization in fractionalorder chaotic systems interacting via a linear time-invariant dynamic coupling, which also is described by fractional derivatives. The obtained results have shown the ability of the dynamic coupling to induce synchronization in the systems, and the strong influence of the integration order on the onset of synchronized behavior has been demonstrated.

Among the observed limitations is that dynamic coupling is sensitive to variations in the integration order of the systems. Therefore, the master and slave systems should have the same integration order. Furthermore, it has been shown that the linearization approach used here to study the local stability of the synchronous solution only provides necessary conditions. Further investigation is needed to derive stronger stability conditions. Perhaps the use of transverse Lyapunov exponents can solve this problem. It remains as future work to extend these results to the bidirectional case and also to the case of networks. Also, it would be interesting to investigate whether any emergent behavior or other types of synchronous behaviors can occur in the coupled systems depending on the integration order of both the systems and the coupling.

Finally, we would like to point out that the results presented here apply to the Caputo definition of fractional derivative. Moreover, these results were obtained using two different numerical approaches, namely, the ABM and the Petrás integrator method. As future work, we plan to compare these results with different fractional operators, such as the Riemann-Liouville operator or the Atangana-Baleanu operator, to name a few.

\section{Data Availability}

The data used to support the findings of the study are included within the article.

\section{Conflicts of Interest}

The authors declare that they have no conflicts of interest.

\section{Acknowledgments}

J. L. E. M. thanks CONACYT for financial support (CVU706850, project: A1-S-26123), J. P. R. for the opportunity to realize a postdoctoral stay at CICESE, and P. A. M. R. for all his teachings and fruitful discussion.

\section{References}

[1] A. Pikovsky, J. Kurths, M. Rosenblum, and J. Kurths, Synchronization: A Universal Concept in Nonlinear Sciences, Cambridge University Press, Cambridge ,UK, 2003.

[2] A. Arenas, A. Díaz-Guilera, J. Kurths, Y. Moreno, and C. Zhou, "Synchronization in complex networks," Physics Reports, vol. 469, no. 3, pp. 93-153, 2008.

[3] G. V. Osipov, J. Kurths, and C. Zhou, Synchronization in Oscillatory Networks, Springer Science \& Business Media, Berlin, Germany, 2007.

[4] O. Boubaker and S. Jafari, Recent Advances in Chaotic Systems and Synchronization: From Theory to Real World Applications, Elsevier, Amsterdam, Netherland, 2018.

[5] A. L. Fradkov, B. Andrievsky, and R. J. Evans, "Controlled synchronization under information constraints," Physical Review E, vol. 78, no. 3, Article ID 036210, 2008. 
[6] H. Nijmeijer and A. Rodriguez-Angeles, Synchronization of Mechanical Systems, World Scientific, Singapore, 2003.

[7] A. L. Fradkov and A. Y. Markov, "Adaptive synchronization of chaotic systems based on speed gradient method and passification," IEEE Transactions on Circuits and Systems I: Fundamental Theory and Applications, vol. 44, no. 10, pp. 905-912, 1997.

[8] A. Ruiz-Silva and J. G. Barajas-Ramírez, "Cluster synchronization in networks of structured communities," Chaos, Solitons \& Fractals, vol. 113, pp. 169-177, 2018.

[9] A. Ruiz-Silva, "Synchronization patterns on networks of pancreatic $\beta$-cell models," Physica D: Nonlinear Phenomena, vol. 416, Article ID 132783, 2021.

[10] A. Ruiz-Silva, H. E. Gilardi-Velázquez, and E. Campos, "Emergence of synchronous behavior in a network with chaotic multistable systems," Chaos, Solitons \& Fractals, vol. 151, Article ID 111263, 2021.

[11] J. Ramirez, A. Arellano-Delgado, and H. Nijmeijer, "Enhancing master-slave synchronization: the effect of using a dynamic coupling," Physical Review E, vol. 98, no. 1, Article ID 012208, 2018.

[12] W. d. Jonge, J. P. Ramirez, and H. Nijmeijer, "Dynamic coupling enhances network synchronization," IFAC-PapersOnLine, vol. 52, no. 16, pp. 610-615, 2019.

[13] J. Pena Ramirez, E. Garcia, and J. Alvarez, "Master-slave synchronization via dynamic control," Communications in Nonlinear Science and Numerical Simulation, vol. 80, Article ID 104977, 2020.

[14] J. M. Munoz-Pacheco, L. C. Lujano-Hernández, C. MuñizMontero et al., "Active realization of fractional-order integrators and their application in multiscroll chaotic systems," Complexity, vol. 2021, Article ID 6623855, 16 pages, 2021.

[15] M. A. Platas-Garza, E. Zambrano-Serrano, J. R. RodríguezCruz, and C. Posadas-Castillo, "Implementation of an encrypted-compressed image wireless transmission scheme based on chaotic fractional-order systems," Chinese Journal of Physics, vol. 71, pp. 22-37, 2021.

[16] X.-Y. Wang and M.-J. Wang, "Dynamic analysis of the fractional-order Liu system and its synchronization," Chaos: An Interdisciplinary Journal of Nonlinear Science, vol. 17, no. 3, Article ID 033106, 2007.

[17] J. L. Echenausía-Monroy, H. E. Gilardi-Velázquez, R. JaimesReátegui, V. Aboites, and G. Huerta-Cuellar, "A physical interpretation of fractional-order-derivatives in a jerk system: electronic approach," Communications in Nonlinear Science and Numerical Simulation, vol. 90, Article ID 105413, 2020.

[18] J. L. Echenausía-Monroy, G. Huerta-Cuellar, R. JaimesReátegui et al., "Multistability emergence through fractionalorder-derivatives in a pwl multi-scroll system," Electronics, vol. 9, no. 6, p. 880, 2020.

[19] C. Wang, "Fractional-order sliding mode synchronization for fractional-order chaotic systems," Advances in Mathematical Physics, vol. 2018, no. 1, 9 pages, Article ID 3545083, 2018.

[20] J. M. Munoz-Pacheco, C. Posadas-Castillo, and E. ZambranoSerrano, "The effect of a non-local fractional operator in an asymmetrical glucose-insulin regulatory system: analysis, synchronization and electronic implementation," Symmetry, vol. 12, no. 9, Article ID 1395, 2020.

[21] S. Yang, C. Hu, J. Yu, and H. Jiang, "Finite-time cluster synchronization in complex-variable networks with fractional-order and nonlinear coupling," Neural Networks, vol. 135, pp. 212-224, 2021.

[22] P.-Y. Xiong, H. Jahanshahi, R. Alcaraz, Y.-M. Chu, J. F. Gómez-Aguilar, and F. E. Alsaadi, "Spectral entropy analysis and synchronization of a multi-stable fractionalorder chaotic system using a novel neural network-based chattering-free sliding mode technique," Chaos, Solitons \& Fractals, vol. 144, Article ID 110576, 2021.

[23] X. Wu, H. Bao, and J. Cao, "Finite-time inter-layer projective synchronization of caputo fractional-order two-layer networks by sliding mode control," Journal of the Franklin Institute, vol. 358, no. 1, pp. 1002-1020, 2021.

[24] A. Sambas, S. Vaidyanathan, I. M. Moroz et al., "A simple multi-stable chaotic jerk system with two saddle-foci equilibrium points: analysis, synchronization via backstepping technique and multisim circuit design," International Journal of Electrical and Computer Engineering, vol. 11, no. 4, pp. 2088-8708, 2021.

[25] M. Tabasi and S. Balochian, "Synchronization of the chaotic fractional-order genesio-tesi systems using the adaptive sliding mode fractional-order controller," Journal of Control, Automation and Electrical Systems, vol. 29, no. 1, pp. 15-21, 2018.

[26] A. Nourian and S. Balochian, "The adaptive synchronization of fractional-order liu chaotic system with unknown parameters," Pramana, vol. 86, no. 6, pp. 1401-1407, 2016.

[27] P. Y. Dousseh, C. Ainamon, C. H. Miwadinou, A. V. Monwanou, and J. B. Chabi Orou, "Chaos in a financial system with fractional order and its control via sliding mode," Complexity, vol. 2021, Article ID 4636658, 15 pages, 2021.

[28] M. Tabasi and S. Balochian, "Synchronization of fractional order chaotic system of sprott circuit using fractional active fault tolerant controller," International Journal of Dynamics and Control, vol. 9, pp. 1-8, 2021.

[29] G. Li, X. Zhang, and H. Yang, "Complexity analysis and synchronization control of fractional-order Jafari-Sprott chaotic system," IEEE Access, vol. 8, pp. 53360-53373, 2020.

[30] P. Anbalagan, E. Hincal, E. Hincal et al., "A Razumikhin approach to stability and synchronization criteria for fractional order time delayed gene regulatory networks," AIMS Mathematics, vol. 6, no. 5, pp. 4526-4555, 2021.

[31] B. Chen and J. Chen, "Razumikhin-type stability theorems for functional fractional-order differential systems and applications," Applied Mathematics and Computation, vol. 254, pp. 63-69, 2015.

[32] J. A. Gallegos and M. A. Duarte-Mermoud, "On the Lyapunov theory for fractional order systems," Applied Mathematics and Computation, vol. 287-288, pp. 161-170, 2016.

[33] S. Liu, W. Jiang, X. Li, and X.-F. Zhou, "Lyapunov stability analysis of fractional nonlinear systems," Applied Mathematics Letters, vol. 51, pp. 13-19, 2016.

[34] Y. Li, Y. Chen, and I. Podlubny, "Stability of fractional-order nonlinear dynamic systems: Lyapunov direct method and generalized Mittag-Leffler stability," Computers \& Mathematics with Applications, vol. 59, no. 5, pp. 1810-1821, 2010.

[35] Y. Li, Y. Chen, and I. Podlubny, "Mittag-Leffler stability of fractional order nonlinear dynamic systems," Automatica, vol. 45, no. 8, pp. 1965-1969, 2009.

[36] E. Ott, "Strange attractors and chaotic motions of dynamical systems," Reviews of Modern Physics, vol. 53, no. 4, pp. 655-671, 1981.

[37] L. Chua, The Genesis of Chua's circuit, Electronics Research Laboratory, College of Engineering, University of California, Berkeley, CA, USA, 1992.

[38] K. Diethelm, The Analysis of Fractional Differential Equations: An Application-Oriented Exposition Using Differential Operators of Caputo Type, Springer Science \& Business Media, Berlin, Germany, 2010. 
[39] I. Podlubny, Fractional Differential Equations: An Introduction to Fractional Derivatives, Fractional Differential Equations, to Methods of their Solution and Some of their Applications, Elsevier, Amsterdam, Netherland, 1998.

[40] E. Zambrano-Serrano, E. Campos-Cantón, and J. M. MuñozPacheco, "Strange attractors generated by a fractional order switching system and its topological horseshoe," Nonlinear Dynamics, vol. 83, no. 3, pp. 1629-1641, 2016.

[41] J. Ramirez, L. Olvera, H. Nijmeijer, and J. Alvarez, "The sympathy of two pendulum clocks: beyond Huygens' observations," Scientific Reports, vol. 6, no. 1, pp. 1-16, 2016.

[42] H. Khalil, Nonlinear Systems, Prentice-Hall, Englewood Cliffs, NJ, USA, 3rd edition, 2002.

[43] I. Echegoyen, V. Vera-Ávila, R. Sevilla-Escoboza, J. H. Martínez, and J. M. Buldú, "Ordinal synchronization: using ordinal patterns to capture interdependencies between time series," Chaos, Solitons \& Fractals, vol. 119, pp. 8-18, 2019.

[44] O. Rössler, "An equation for continuous chaos," Physics Letters A, vol. 57, no. 5, pp. 397-398, 1976.

[45] S. Bhalekar and M. Patil, "Singular points in the solution trajectories of fractional order dynamical systems," Chaos: An Interdisciplinary Journal of Nonlinear Science, vol. 28, no. 11, Article ID 113123, 2018.

[46] K. Diethelm, N. J. Ford, and A. D. Freed, "A predictor-corrector approach for the numerical solution of fractional differential equations," Nonlinear Dynamics, vol. 29, no. 1/4, pp. 3-22, 2002.

[47] A. Jafari, I. Hussain, F. Nazarimehr, S. M. R. H. Golpayegani, and S. Jafari, "A simple guide for plotting a proper bifurcation diagram," International Journal of Bifurcation and Chaos, vol. 31, no. 1, Article ID 2150011, 2021.

[48] L. Chua, M. Komuro, and T. Matsumoto, "The double scroll family," IEEE Transactions on Circuits and Systems, vol. 33, no. 11, pp. 1072-1118, 1986.

[49] R. Hilborn, Chaos and Nonlinear Dynamics: An Introduction for Scientists and Engineers, Oxford University Press on Demand, Oxford, UK, 2000.

[50] I. Petráš, "Fractional-order chaotic systems," in FractionalOrder Nonlinear Systems, pp. 103-184, Springer, Berlin, Germany, 2011.

[51] H. E. Gilardi-Velázquez and E. Campos-Cantón, "Nonclassical point of view of the Brownian motion generation via fractional deterministic model," International Journal of Modern Physics C, vol. 29, no. 3, Article ID 1850020, 2018. 